\title{
A structural view of the antibiotic degradation enzyme NDM-1 from a superbug
}

\author{
Yu Guo ${ }^{1,3^{*}}$, Jing Wang ${ }^{1,3^{*}}$, Guojun Niu ${ }^{1,3}$, Wenqing Shui ${ }^{1,3}$, Yuna Sun ${ }^{4}$, Honggang Zhou ${ }^{1,3}$, Yaozhou Zhang ${ }^{1}$, \\ Cheng Yang ${ }^{1,3}$, Zhiyong Lou ${ }^{2 \bowtie}$, Zihe Rao ${ }^{1,2,3,4} \bowtie$ \\ ${ }^{1}$ High-throughput Molecular Drug Discovery Center, Tianjin Joint Academy of Biotechnology and Medicine, Tianjin 300071, \\ China \\ 2 Laboratory of Structural Biology, Tsinghua University, Beijing 100084, China \\ ${ }^{3}$ College of Life Science, Nankai University, Tianjin 300074, China \\ ${ }^{4}$ National Laboratory of Macromolecules, Institute of Biophysics, Chinese Academy of Sciences, Beijing 100101, China \\ $\triangle$ Correspondence: louzy@xtal.tsinghua.edu.cn (Z. Lou), raozh@xtal.tsinghua.edu.cn (Z. Rao)
}

Received March 22, 2011 Accepted April 10, 2011

\begin{abstract}
Gram-negative Enterobacteriaceae with resistance to carbapenem conferred by New Delhi metallo- $\beta$-lactamase 1 (NDM-1) are a type of newly discovered antibioticresistant bacteria. The rapid pandemic spread of NDM-1 bacteria worldwide (spreading to India, Pakistan, Europe, America, and Chinese Taiwan) in less than 2 months characterizes these microbes as a potentially major global health problem. The drug resistance of NDM-1 bacteria is largely due to plasmids containing the blaNDM-1 gene shuttling through bacterial populations. The NDM-1 enzyme encoded by the blaNDM-1 gene hydrolyzes $\beta$-lactam antibiotics, allowing the bacteria to escape the action of antibiotics. Although the biological functions and structural features of NDM-1 have been proposed according to results from functional and structural investigation of its homologues, the precise molecular characteristics and mechanism of action of NDM-1 have not been clarified. Here, we report the threedimensional structure of NDM-1 with two catalytic zinc ions in its active site. Biological and mass spectroscopy results revealed that $D$-captopril can effectively inhibit the enzymatic activity of NDM-1 by binding to its active site with high binding affinity. The unique features concerning the primary sequence and structural conformation of the active site distinguish NDM-1 from other reported metallo- $\beta$-lactamases (MBLs) and implicate its role in wide spectrum drug resistance. We also discuss the molecular mechanism of NDM-1 action and its
\end{abstract}

essential role in the pandemic of drug-resistant NDM-1 bacteria. Our results will provide helpful information for future drug discovery targeting drug resistance caused by NDM-1 and related metallo- $\beta$-lactamases.

KEYWORDS New Delhi metallo- $\beta$-lactamase 1 (NDM1), drug resistance, crystal structure, drug discovery

\section{INTRODUCTION}

The growing increase in the rate of antibiotic resistance is a major cause for concern with both non-fermenting bacilli and isolates of, e.g., the Enterobacteriaceae, Acinetobacter baumannii, Pseudomonas aeruginosa, and pneumoniacausing bacteria (Livermore, 2009; Moellering, 2010). Methicillin- and vancomycin-resistant gram-positive bacteria and multidrug-resistant gram-negative bacteria cannot be effectively treated with proven therapeutics and pose the greatest risk to public health (Heddini et al., 2009; Baiden et al., 2010; Kumarasamy et al., 2010). Since the initial discovery in 2009 of a Swedish patient who traveled to New Delhi, India and acquired a urinary tract infection caused by a carbapenemresistant Klebsiella pneumoniae strain (Yong et al., 2009), hundreds of instances of infection by this antibiotic-resistant bacteria have been reported (Baiden et al., 2010; Moellering, 2010; Struelens et al., 2010; Walsh, 2010; Chihara et al., 2011). What's more, NDM-1 positive bacteria were found from drinking water and sewage water in India recently (Walsh et al., 2011). The first discovery of NDM-1 outside hospital environment raises serious concerns about this wide

\footnotetext{
*These authors contributed equally to the work.
} 
spectrum antibiotic resistant-bacteria.

The increase in antibiotic resistance of gram-negative bacteria was first designated by transmissible gene elements on plasmids that can rapidly spread through bacterial populations (Kumarasamy et al., 2010). Using molecular biology, the gene carried by the mobile plasmid that caused the multidrug-resistance was identified as blaNDM-1, which encodes the New Delhi metallo- $\beta$-lactamase 1 (NDM-1) enzyme (Yong et al., 2009; Kumarasamy et al., 2010).

NDM-1 belongs to the Class B metallo- $\beta$-lactamase (MBL) superfamily, which contains enzymes that hydrolyze $\beta$ lactams and plays a key role in antibiotic-resistant bacterial infections. Conversely, class A, C and D $\beta$-lactamases are structurally characterized as serine $\beta$-lactamases (Bebrone, 2007; Shimada et al., 2010). The MBL superfamily is a large clade of proteins composed of 20 families (e.g., glyoxalase II, cyclase, arylsulfatase, alkylsulfatase, flavoprotein, and ribonuclease) (Daiyasu et al., 2001), which was first discovered by Sabbath and Abraham (1966) in a Bacillus cereus isolate demonstrating in vitro cephalosporinase activity (Abraham and Chain, 1988).

Structurally, MBL members share a so-called highly conserved MBL fold, which is characterized as a four-layered $\beta$-sandwich with two mixed $\beta$-sheets flanked by $\alpha$-helices. An additional distinct structural feature of MBLs is that one or two catalytic zinc or iron ions bind to the active site and are necessary for the catalytic reaction (Bebrone, 2007). Interestingly, the substrate specificity of different MBLs is not associated with the common MBL fold but achieved by the structures flanking the active site (Bebrone, 2007; Shimada et al., 2010). MBLs specifically cleave the amide bond of the $\beta$ lactam ring, thus inactivating the antibiotic (Bebrone, 2007). The functional feature distinguishing MBLs from other $\beta$ lactamases is that MBLs can degrade all classes of $\beta$-lactams except monobactams (Bebrone, 2007). Therefore, MBLcontaining bacteria raise antibiotic resistance concerns worldwide.

Because bacterially encoded MBLs hydrolyze antibiotics and thus cause drug resistant bacterial infections, it is obvious that specially designed antibiotics that are poor substrates for all bacterially encoded MBLs can overcome the drug resistance induced by MBLs. However, as currently reported, bacterially encoded MBLs display wide substrate specificity, making the design of special antibiotics a difficult problem to solve. Alternatively, isolating $\mathrm{MBL}$ inhibitors may be more practical. However, while several $\beta$-lactamase inhibitors have been used clinically (e.g., tazobactam) (Bauernfeind et al., 1998), all such reagents are currently characterized as class A $\beta$-lactamase (e.g., serine $\beta$-lactamase) inhibitors. Thus, clinically useful inactivators of MBLs (Class B $\beta$-lactamases) are still lacking.

The NDM-1 enzyme was first characterized as an MBL due to its high similarity in amino acid sequence with several reported bacterially encoded MBLs (Bebrone, 2007), indicating similarity with their three-dimensional (3-D) structures and molecular mechanism of action. However, detailed structural information and the precise molecular mechanism of NDM-1 activity remain unclear. Here, we report the first crystal structure of NDM-1, which presents a typical MBL fold with two catalytic zinc ions in its active site. Biological and mass spectroscopy results revealed that D-captopril can inhibit the enzymatic activity of NDM-1 in vitro by binding to the NDM-1 active site with high affinity. Moreover, the role of residues flanking the active site was further investigated by mutagenesis. Finally, we discuss the molecular mechanism of NDM-1 action and its essential role in the pandemic of NDM-1 bacteria. Our results are helpful for future drug discovery targeting drug-resistant bacteria encoding NDM-1 and related MBLs.

\section{RESULTS}

\section{The overall structure of NDM-1}

The crystal structure of the 24-kDa truncated NDM-1 (from Gly47 to Arg270 with six additional N-terminal residues introduced by the expression vector) was solved by the molecular replacement method and refined to $2.5 \AA$ resolution, resulting in a final $R_{\text {work }}$ value of $23.4 \%\left(R_{\text {free }}=26.7 \%\right)$. The crystal belongs to the $P 3_{1}$ space group, and there are two NDM-1 molecules per asymmetric unit with a Matthews coefficient of $2.2 \AA^{3} / \mathrm{Da}$ (corresponding to a $43 \%$ solvent content) (Matthews, 1968). The first $46 \mathrm{~N}$-terminal residues in the native protein were truncated for better expression and purification. Because these $\mathrm{N}$-terminal residues are not involved in enzymatic activity, they do not likely affect the folding and enzymatic characteristics of NDM-1. Gel filtration and analytical ultracentrifugation (AUC) (data not shown) revealed that NDM-1 exists as a monomer in solution, which is consistent with our crystallographic results (contact surface of $\sim 220 \AA^{2}$, which is $10 \%$ of the total molecular surface of $2900 \AA^{2}$ ). Because reported NDM-1 homologues function as monomers (Yong et al., 2009), we speculate that the monomer is the functional unit of NDM-1 in vitro and in vivo. Moreover, two bound metal ions were revealed by the anomalous difference map and further confirmed by the atomic absorption spectrum to be zinc ions. Because there is no zinc added during either purification or crystallization, these two zinc ions must biologically bind to NDM-1 and possibly have functional implications.

We have found that NDM-1 enzyme possesses a compact globular structure of dimensions $50 \AA \times 40 \AA \times 40 \AA$ and displays the canonical $\alpha \beta \beta \alpha$ sandwich architecture of the MBL superfamily with two central anti-parallel $\beta$-sheets flanked by two pairs of $\alpha$-helices (Fig. 1A). Structure comparison via Secondaty Structure Matching (SSM) (Krissinel and Henrick, 2004) uncovered several structural homologues from the MBL superfamily. The most significant 
A

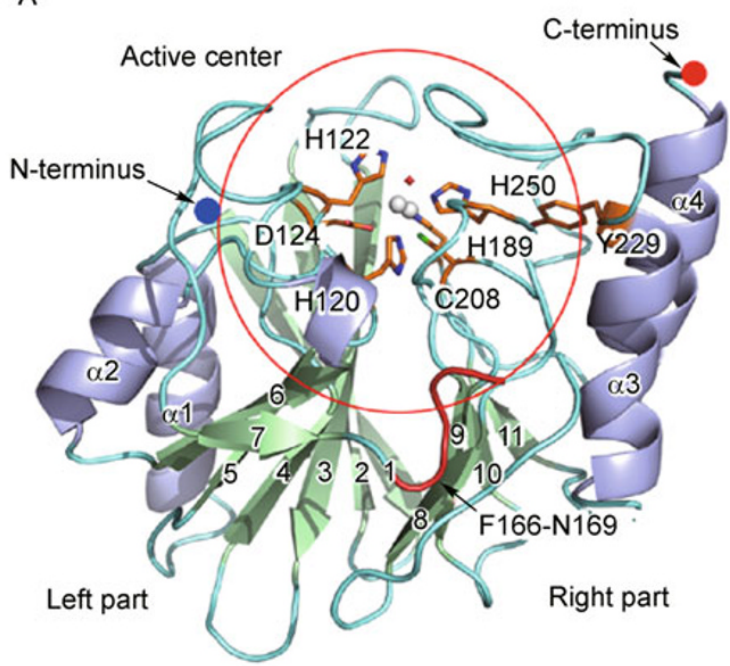

C

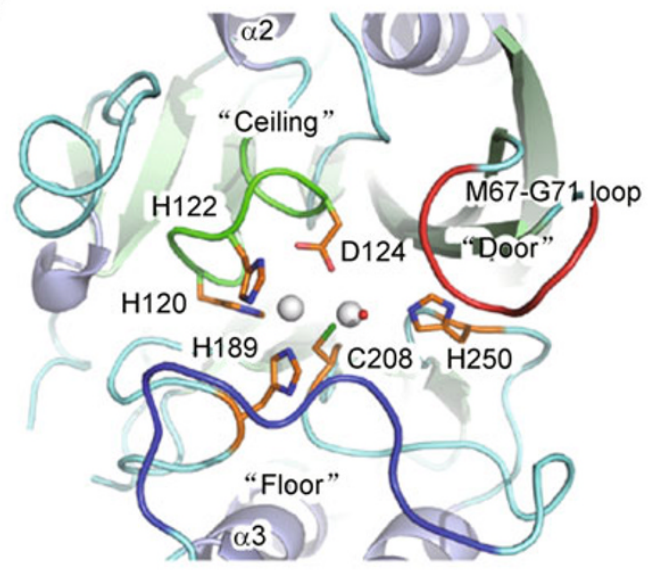

B

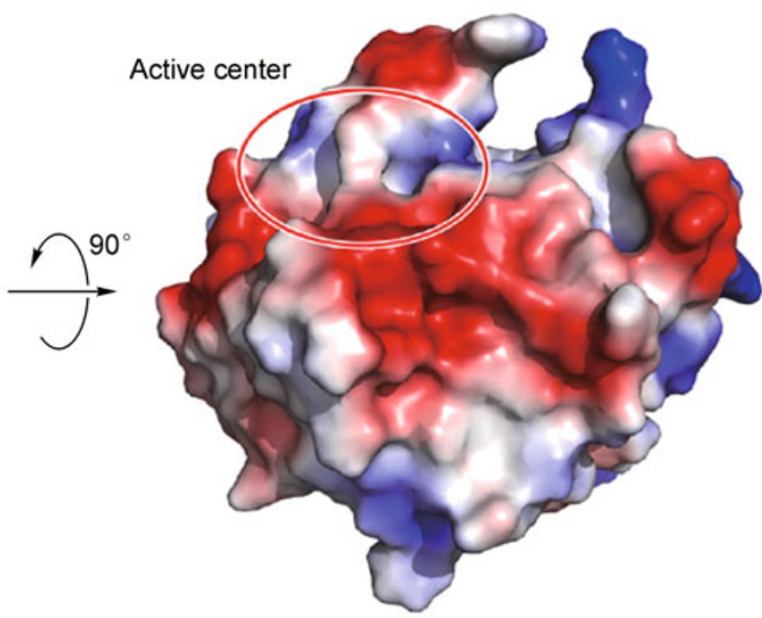

D

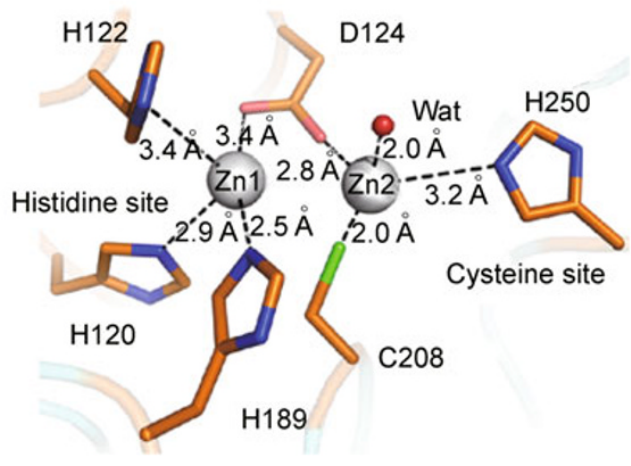

Figure 1. Crystal structure of NDM-1. (A) Overall ribbon diagram of the crystal structure of NDM-1. The $\alpha$-helices are colored light blue, while the $\beta$-strands and the loop regions are green and cyan, respectively. The catalytic zinc ions and bound solvent molecule are presented as spheres, while the key residues in the active center are shown as colored sticks. All secondary structural elements and key residues have been labeled; the $\mathrm{N}$ - and $\mathrm{C}$-terminus and the active center are marked. The active site is framed with a red circle. Try229 and Phe166-Asn169, which are previously reported to be unique in NDM-1 compared to other reported MBLs, are labeled out with red. (B) Electrostatic potential surface of NDM-1 (vertical orientation). Red = negative and blue = positive. (C) The loop regions (Met67-Gly71) acting as the "Door," Thr119-Met126 acting as the "Ceiling," and Ser217-Asp225 acting as the "Floor" are colored red, green, and blue, respectively. The key residues and secondary structures are labeled. (D) Enlarged view of the active center of NDM-1. Two catalytic zinc ions and bound solvent molecule are shown as spheres, and key residues are shown as colored sticks. The interaction between the residues and bound metal ions and the distances are labeled.

matches, based on the lowest root mean square (r.m.s.) deviation values, include $\mathrm{Bc} / \mathrm{l}$ (PDB code: $1 \mathrm{BMC}, 32 \%$ amino acid identity, r.m.s.d of $\mathrm{Ca}=1.3 \AA$ ) (Carfi et al., 1995), IMP-1 (PDB code: 1DD6, 33\% amino acid identity, r.m.s.d of $\mathrm{Ca}=$ $1.6 \AA$ ) (Concha et al., 2000), BlaB (PDB code: 1M2X, 25\% amino acid identity, r.m.s.d of $\mathrm{Ca}=1.7 \AA$ ) (García-Saez et al., 2003a), VIM-4 (PDB code: 2WRS, 36\% amino acid identity, $r$. m.s.d of $\mathrm{Ca}=1.2 \AA$ ) (Lassaux et al., 2010), VIM-2 (PDB code:
$1 \mathrm{KO} 2,36 \%$ amino acid identity, r.m.s.d of $\mathrm{Ca}=1.2 \AA$ ) (GarciaSaez et al., 2008) of the subclass B1 MBLs, and CphA (PDB code: $2 \mathrm{GKL}, 25 \%$ amino acid identity, r.m.s.d of $\mathrm{Ca}=1.7 \AA$ ) (Garau et al., 2005) of the B2 subclass. Although the sequence identity of NDM-1 with the B3 subclass MBL FEZ-1 (PDB code: 2X8I) (García-Saez et al., 2003b) is $<10 \%$, the topologies of the structural superimpositions are remarkably similar (Fig. 2). 
A
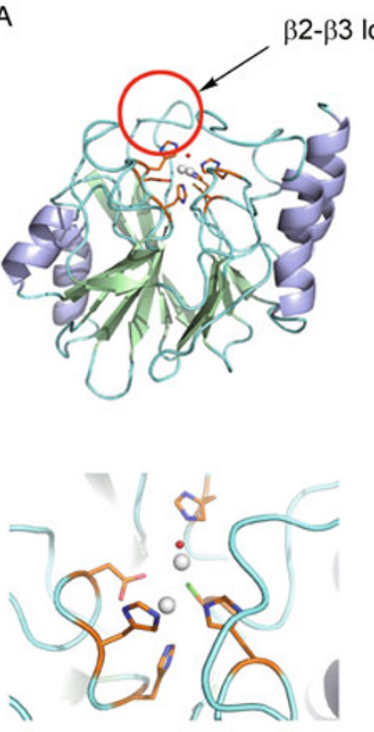

NDM-1
2- $\beta 3$ loop
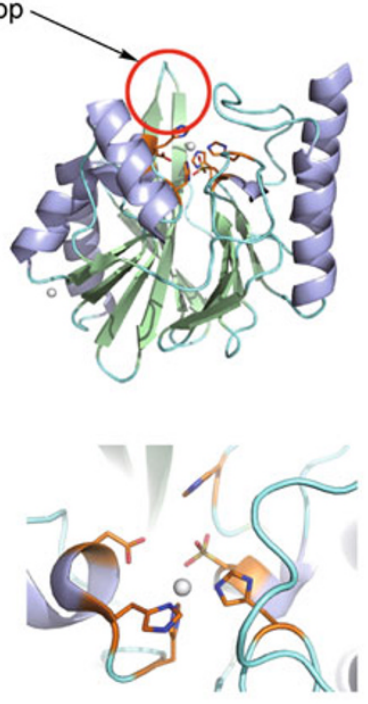

VIM-2
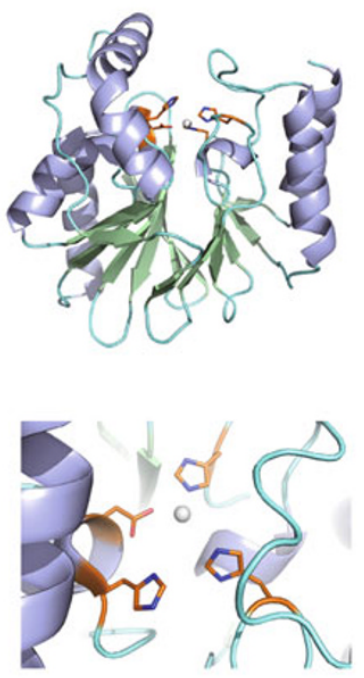

CphA
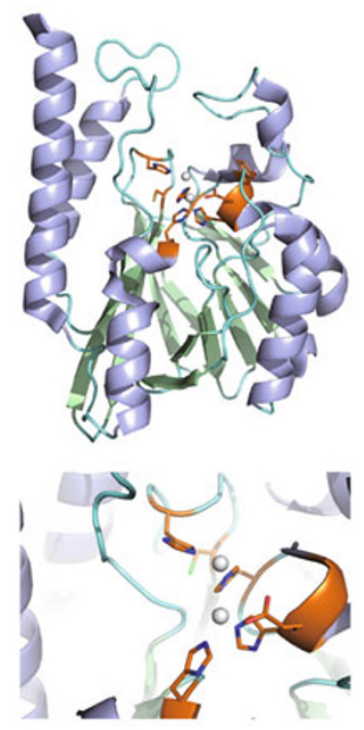

FEZ-1

B
$47 \stackrel{\beta 1}{\rightarrow}$
${ }_{56} \stackrel{\beta 2}{\longrightarrow}$
$\stackrel{\beta 2}{\longrightarrow}$
$\underset{86}{\stackrel{\beta 4}{\longrightarrow}}$
96
$\alpha 1$
$\frac{\beta 5}{116}$

NDM-1
IMP-1
VIM-2
BlaB
CphA

.... GDLVERQLAPNVWQHTSYLDMPGE GAVAS NंGLIVRDG GRVLVVDTAWTTDQTAQILNWITREINLPVALAY .....AESLP DLKIEKLDEGVYVHTSFEEVNGWGVVPKHGLVVLVNAEAYLIDTPFTAKDTEKLVTWFVERG. YKIKGS EYPTVSEIPVGEVRLYQIADGVWSHIATQSFDG. AVYPS NGL IVRDGDELLLIDTAWGAKNTAAL LAEIEKQIGLPVTRAV $\ldots \ldots \ldots \cdots$
$\ldots \ldots$
$\ldots$

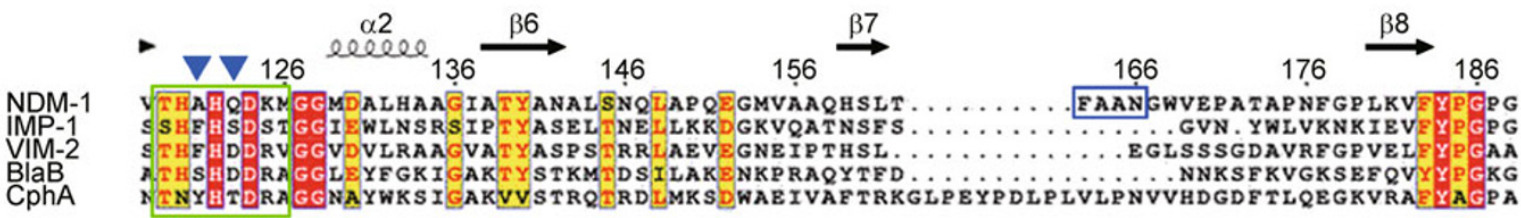
$\Delta \Delta \Delta$

$$
\underset{196}{\stackrel{\beta 9}{\longrightarrow}} \underset{206}{\stackrel{\beta 10}{\longrightarrow}}
$$

$226 \quad 236$

$\underset{246}{\stackrel{\beta 11}{\longrightarrow}}$

256 ereecer

NDM-1

IMP-1

VIM-2

BlaB

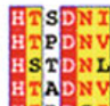

IIVIDGTDIA

\section{6}

AD TEHYAASARAFGAAFP KASMIVMS

VWLPERKI L
VYVPSASVL
VWEPEKVL

LIKDSKAKS LENLG

KAKSLGNLGDA

CAIYELSRTSAGNVADAD LAEWPTSIERIQQHYPEAQFVIPGHGLPGGLDLLKHTTIVVKAH HDPDGIFUYFEDEQVLYGNCILKEK. .

$\Delta$

$\Delta$

宷

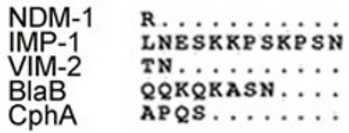

Figure 2. Structure and primary sequence comparison of NDM-1 with its homologues. (A) Structural comparison of NDM-1, VIM2 (PDB code: 1KO2), CphA (PDB code: 1DD6) and FEZ-1 (PDB code: 1K07). All structures are shown with the same orientation and colored by their secondary structure with the same color scheme as in Fig. 1. The bound metal ions and key residues are also shown. (B) Primary sequence alignment of NDM-1, IMP-1, VIM-2, BlaB, and CphA. Secondary structure elements of NDM-1 are indicated on top of the alignment; $\alpha$-helices and $\beta$-strands are presented as curves and arrows, respectively. The residue numbers at the top refer to the blaNDM-1 gene. The residues coordinating zinc ions are marked with upright red triangles; Ala121 and Gln123 are marked with inverted blue triangles. The loop regions, which are assigned as the ceiling, floor and door of the active site, are framed in the color of green, blue, and red, respectively. Tyr229 and Phe166-Asn169 are also boxed and highlighted. 
The left portion of the NDM-1 molecule consists of two $\alpha$ helices and seven anti-parallel $\beta$-strands, while the right subdomain is formed by the remaining two $\alpha$-helices ( $\alpha 3$ and $\alpha 4)$ and four anti-paralleled $\beta$-strands ( $\beta 8-\beta 11)$. A previous report suggests that the NDM-1 possesses additional residues from Phe163 to Asn166, which locate at the loop region connecting left and right subdomains, and are not observed in other reported MBLs. It indicates these residues might be unique for the structure and function of NDM-1 (Yong et al., 2009). However, in the crystal structure of NDM-1, these four residues were identified to locate far away from the active site with a distance of $\sim 20 \AA$ (Fig. 1A), indicating that these residues may not be directly involved in the catalytic reaction of NDM-1. The two catalytic zinc ions, coordinated by His120, His122, Asp124, His189, Cys208 and His250, and located at the external edge of the $\beta \beta$ sandwich, are bridged by the side chain of Asp124 and act as catalytic ions. The loop region containing Met67-Gly71, connecting $\beta 2-\beta 3$ and characterized to play a crucial role in MBL substrate and inhibitor binding, was identified by unambiguous electron density, while the equivalent portion in the native Bc/l enzyme structure was totally disordered (though stabilized after binding to substrate or inhibitor) (Carfi et al., 1995).

Further, the loop regions connecting $\beta 5-\alpha 2$ and $\beta 10-\alpha 4$ clamp this active site, acting as the "ceiling" and "floor," respectively (Fig. 1C). In the NDM-1 crystal structure, the stereochemistry of Phe70, Asp90, Asn110 and Lys216 are located in the disallowed areas of the Ramachandran plot (Laskowski et al., 1993). Unlike Asn110 and Lys216, which have unclear electron density, Asp90 is well defined at the connecting portion of $\beta 4-\alpha 1$ and stabilizes the loop region between $\beta 5-\alpha 2$ (which defines the $\mathrm{Zn} 1$ binding site) through hydrogen bonds with Thr119 and Lys124. However, Phe70 is in the classical $\beta 2-\beta 3$ loop and may play a key role in substrate/inhibitor binding. Here, it is forced to adopt a strained conformation in NDM-1 and likely adopts a more canonical conformation when NDM-1 is bound to substrate.

\section{NDM-1 active site}

The active site of the NDM-1 enzyme is distinctly characterized by two bound zinc ions, indicating a two-ion catalytic mechanism that is characteristic of the MBL superfamily (Bebrone, 2007). In the NDM-1 structure, the active site centered around the two zinc ions is surrounded by His120, His122, Asp124, His189, Cys208 and His250. One zinc ion (Zn1) displays tetrahedral coordination formed by His120His122-His189 and one hydroxyl of Asp124 (i.e., located in what is referred to as the histidine site in the MBL superfamily (Bebrone, 2007)), and the second zinc ion ( $\mathrm{Zn} 2)$ has a trigonal-pyramidal coordination sphere involving one hydroxyl of Asp124-Cys208-His250 (i.e., the cysteine site) (Fig. 1D) (Bebrone, 2007). The two zinc ions contact each other with a distance of $3.2 \AA$ and are bridged by the side chain of Asp124.
In contrast, the sole metal ion of the Bc/l, VIM-2 and SPM-1 enzymes is located only in the histidine site (Carfi et al., 1995; García-Saez et al., 2008).

The results of NDM-1 enzymatic assays demonstrate that EDTA can inhibit the activity of NDM-1 in vitro by chelating the catalytic zinc ions (Fig. 3A). A solvent molecule is bound to the zinc ion in the "cysteine" site and the $\mathrm{Zn}-\mathrm{O}$ distance of 2.0 $\AA$ indicates a bound hydroxide which may serve as the attacking nucleophile on the carbonyl carbon of the $\beta$-lactam ring (Fig. 1D). However, a similar hydroxide group in the FEZ1 structure bridges the two zinc ions (García-Saez et al., $2003 \mathrm{~b}$ ) instead of binding the zinc ion at the cysteine site as in NDM-1. We speculate that this difference is related to different enzymatic mechanisms used by the enzymes. Nonetheless, elucidating the precise mechanism of NDM-1 action requires further investigation.

The NDM- 1 active site is a deep cavity that is formed by the loop regions between $\beta 5-\alpha 2$ and $\beta 10-\alpha 4$, in which the catalytic zinc ions are deeply buried. This cavity is clamped by the loop regions between Thr119-Met126 and Ser217-Asp225 (i.e., the ceiling and floor), while the Met67-Gly71 loop acts as a doorkeeper to close the site during substrate binding and open it for product release via conformational changes (Fig. 1C). Sequence alignments suggest that the ceiling and floor are largely conserved in both class B1 and B2 MBLs. However, the Met67-Gly71 loop in NDM-1 has less similarity with other reported MBL structures.

In subclass B1 MBLs, the $\mathrm{N}$-terminus includes a loop region composed of residues 61-65 (numbered as in the $\mathrm{Bcll}$ sequence (Carfi et al., 1995)) that is absent in subclass $B 2$ and B3 MBLs, which also distinguishes the B2 and B3 subclasses from the B1 subclass (Bebrone, 2007). This loop region can interact with substrate or inhibitor molecules that possess hydrophobic side chains, form a tunnel-shaped cavity at the active site groove, and thus block the molecule in the active site (Bebrone, 2007). Biological results demonstrate that deletion of this loop seriously affects the enzymatic activity by weakening substrate binding by the enzyme (with the exception of imipenem). Although this loop region is totally disordered in the native BlaB structure (García-Saez et al., 2003a), it is unambiguously characterized by electron density in our NDM-1 structure and composed of Met67-Gly71.

However, this loop region in NDM-1 adopts a curved conformation and covers the active site instead of the extended conformation (even if it is not as disordered as in the BlaB structure) in other reported native subclass B1 MBLs. This suggests that this loop region has more mobility during substrate binding, and NDM-1 may exhibit a different substrate interacting mode and enzymatic mechanism than related MBLs (Fig. 2). Moreover, the movement of this loop is generally considered to be related to the interaction of the side chain of Trp64 (numbered as in the IMP-1 structure (Concha et al., 2000)) with the hydrophobic side chain of the 
A

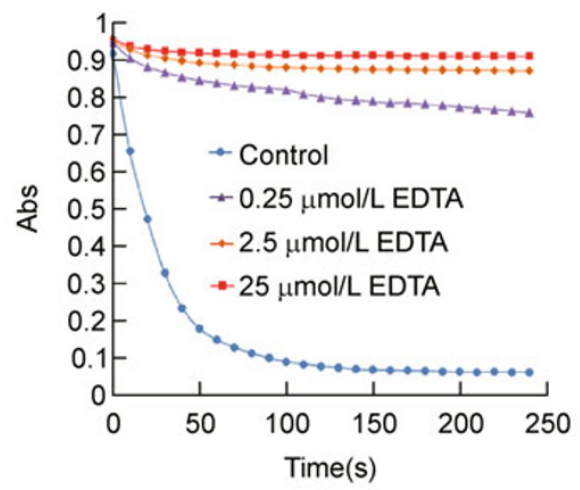

C

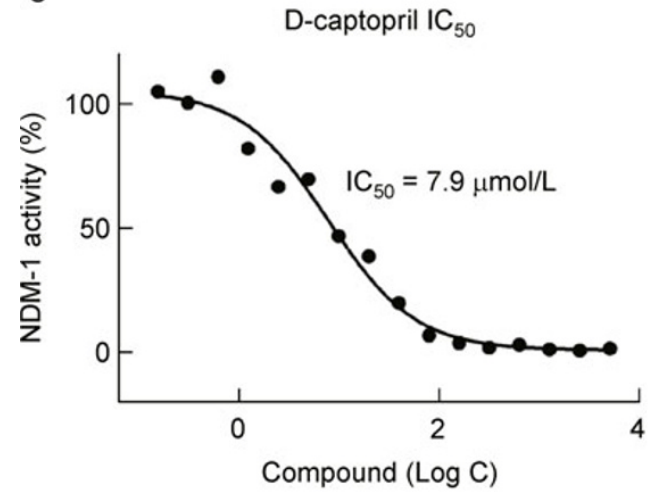

$\mathrm{E}$

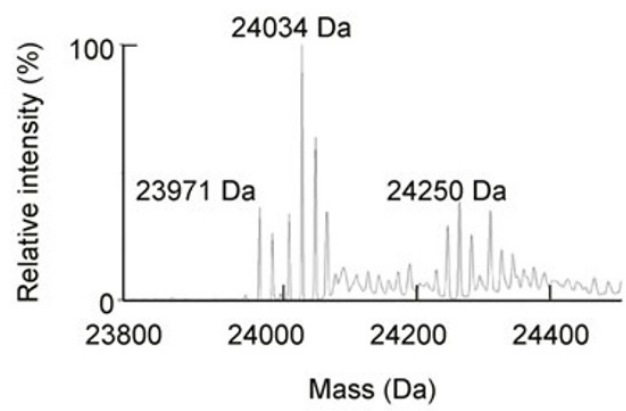

B

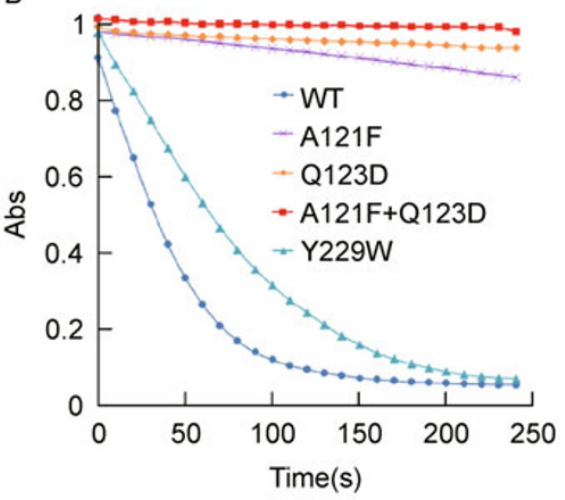

D

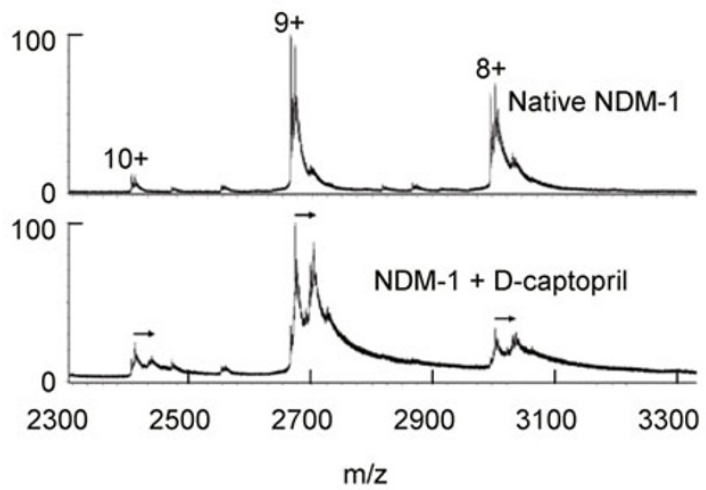

$\mathrm{F}$

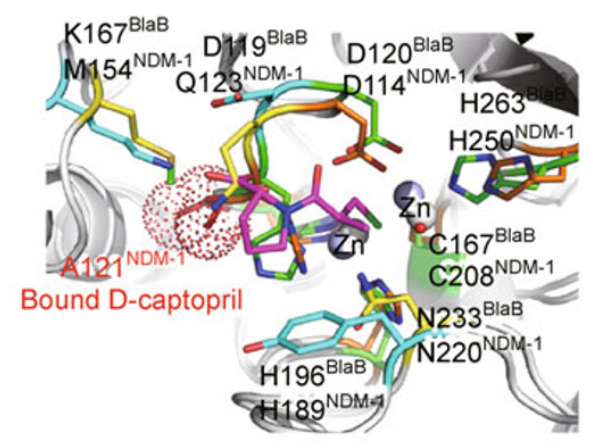

Figure 3. Enzymatic study and inhibitory mechanism. (A) Enzymatic assays of NDM-1 inhibited by various concentrations of EDTA. (B) Imipenem hydrolysis activity of wild type NDM-1 and mutants monitored at $300 \mathrm{~nm}$. (C) Inhibition of NDM-1 enzymatic activity by D-captopril. (D) Electrospray mass spectrometry results of NDM-1 bound to D-captopril. The charge states of individual protein peaks are annotated in the upper panel. Arrows indicate the mass shift for each charge state of the complex formed between the protein and the ligand. (E) Deconvoluted mass spectrum of NDM-1 in complex with D-captopril. Mass measurement was based on the raw spectrum shown in the bottom panel of Fig. $1 \mathrm{E}$. The measured mass of the free protein is $23,971 \mathrm{Da}$, while the peak at $24,034 \mathrm{Da}$ indicates the free protein bound to a zinc ion. The third peak at 24,250 Da is the result of D-captopril binding to NDM-1 (capturing $\left.\mathrm{Zn}^{2+}\right)$. The mass shift $(216 \mathrm{Da})$ is close to the atomic mass of the ligand (217 Da). (F) Modeled complex structure of NDM-1 and D-captopril based on the structural superimposition between NDM-1 and IMP-1. The secondary structure of NDM- 1 is shown as a grey ribbon, the modeled bound D-captopril is shown as sticks in purple, the key residues bound to metal ions in NDM-1 and IMP-1 are shown as golden and green sticks, and the residues that may also participate in D-captopril binding in NDM-1 and IMP-1 are shown as yellow and cyan sticks.

substrate (Moali et al., 2003). Unexpectedly, the equivalent position is Phe70 in NDM-1 (Fig. 2B), which has a similar hydrophobic side chain and suggests a similar function.
However, the same position is not found in the amino acid sequences of VIM-2 and BlaB (B1 subclass) or CphA (B2 subclass), indicating that the mechanism of NDM-1 action 

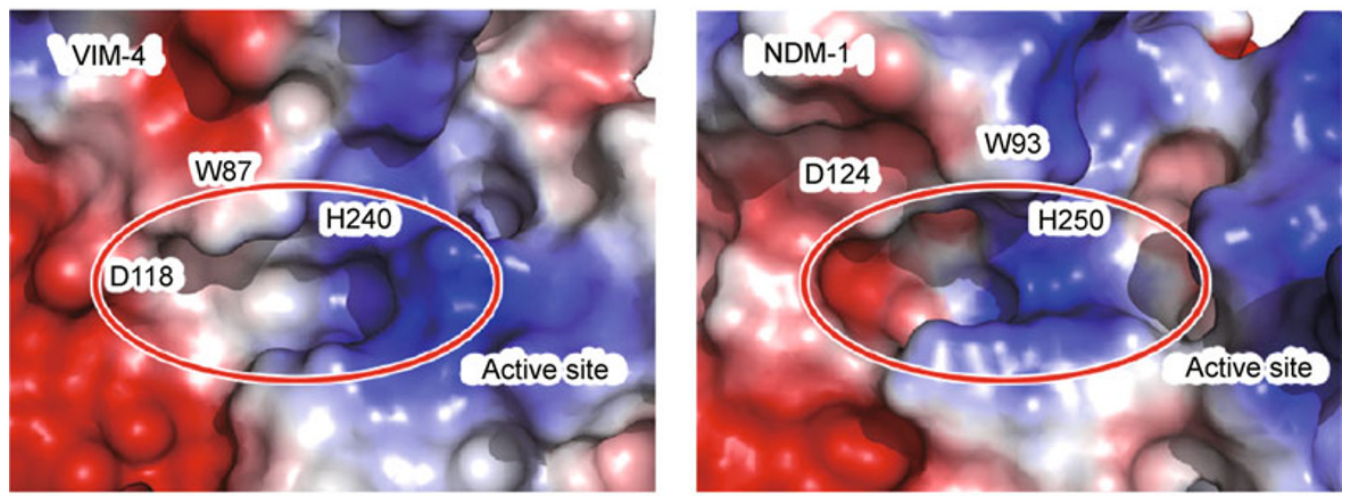

Figure 4. Comparison of potential surface of VIM-4 and NDM-1. The active sites of VIM-4 (left panel) and NDM-1 (right panel) are shown as potential surface. The active sites are circled, and residues that form the deep hydrophilic hole are labeled.

may be more similar to IMP-1 than to other MBLs. Furthermore, although Tyr229 in NDM-1 was considered to be a unique feature of NDM-1 from primary sequence analysis compared to the universal conserved tryptophan (Yong et al., 2009), this residue is obstructed from the active site by the "floor" region, indicating that Tyr229 may not directly participate in enzymatic reaction of NDM-1 (Fig. 1A). Nonetheless, hydrogen bonds formed by the side chain of Tyr229 with Leu209 and Gly188 located at the door and floor regions indicate that Tyr229 should be crucial for stabilizing the conformation of active site of NDM-1. Interestingly, although NDM-1 is identified to be a Class B1 MBL, Tyr229 of NDM-1 is not conserved with other reported Class B1 MBLs but shows high similarity with Class B3 MBL, FEZ-1 (Fig. 2B) (Yong et al., 2009). Our structure shows that Tyr229 is on the periphery of the active site and may not directly participate in the enzymatic action of NDM-1, as confirmed by the minor effect of the Y229W mutation (Fig. 3B), suggesting the conservation of this residue and its functional role in catalytic reaction in MBL superfamily should need further investigation.

Sequence alignment suggests that NDM-1 possesses a unique HAHQD motif compared to the universally conserved HFHDD motif in other MBLs (Yong et al., 2009). Although the substitutions appear to be quite conservative, the Q123D and A121F mutations decreased the imipenem hydrolysis activity of NDM-1 by $98.4 \%$ and $96.4 \%$, respectively, while the Q123D/A121F double mutant retained only $0.7 \%$ of the wildtype activity (Fig. 3B), indicating a possible evolutionary advantage of NDM-1. Furthermore, the deep cavity of NDM-1 active site provides a larger volume than that of other reported MBLs, e.g. VIM-4, with an extraordinary hydrophilic hole surrounded by Trp93, Gln123, Asp124 and His250, and thus could provide more space for NDM- 1 to adopt its substrate in the active site than other reported MBLs (Fig. 4). As NDM-1 was reported to show high binding affinity to most cephalosporins, in particular, to cefuroxime, cefotaxime, and cepha- lothin and to the penicillins, which is unusual for an MBL (Yong et al., 2009), we speculate that the larger space in NDM-1 active site might contribute to the wide spectrum antibiotics substrate selectivity of NDM-1. Taken together, these results therefore indicate that the spatial conformation and potential feature of active site of NDM-1 should be crucial for its activity.

\section{Therapeutic implications for targeting drug-resistant NDM-1 bacteria}

Although the metal chelator EDTA totally abolished the enzymatic activity of NDM-1 (Fig. 3A), which is consistent with the crucial role of bound catalytic metal ions in the mechanism of action of MBL superfamily enzymes (Garau et al., 2005), it is apparent that compounds with similar inhibitory mechanisms will have no clinical significance. Nonetheless, a few of potential clinical reagents that specifically inhibit MBL activity have been introduced clinically, including thioester derivatives, sulfonyl hydrazones, tricyclic natural products, succinic acid derivatives, biphenyl tetrazoles carbapenem and penicillin derivatives, degradation products of cephalosporins, thioesters, thiomandelic acid, captopril, benzohydroxamic acid derivatives and pyridine carboxylates (Bebrone, 2007). Among these compounds, D-captopril is widely used to investigate the enzymatic and inhibitory mechanisms of MBL proteins, and it is characterized as a potential clinical reagent for the treatment of drug-resistant bacterial infections (Concha et al., 2000).

Unexpectedly, D-captopril also displayed a high inhibitory effect on NDM-1 enzymatic activity in vitro, with an $\mathrm{IC}_{50}$ value of $7.9 \mu \mathrm{mol} / \mathrm{L}$ (Fig. 3C). Though our attempts to generate a complex structure of NDM-1 bound to D-captopril failed due to poor diffraction after soaking, mass spectroscopy results distinctly revealed that D-captopril could bind to NDM-1 enzyme (Fig. 3D and 3E). Interestingly, L-captopril (which is used clinically to treat hypertension, congestive heart failure, 
and renal syndromes as an angiotensin-converting enzyme (ACE) inhibitor and is known to inhibit several MBLs in vitro) (Antony et al., 2002) inhibited NDM-1 activity with an $\mathrm{IC}_{50}$ value of $202.0 \mu \mathrm{mol} / \mathrm{L}$. This suggests that the pyrrolidine motif of L- and D-captopril is crucial for its binding to NDM-1.

Because NDM-1 shares a high amino acid sequence and structural conservation (especially at the active site) with other MBLs, we believe that molecular modeling of NDM-1 bound to D-captopril could provide useful information to understand the enzymatic and inhibitory mechanisms of NDM-1. Garcia-Saez et al. (2008) reported a high resolution crystal structure of BlaB in complex with D-captopril (PDB code: $1 \mathrm{M} 2 \mathrm{X})$, demonstrating a clear inhibitory mechanism for D-captopril against BlaB, a Class B $\beta$-lactamase (GarcíaSaez et al., 2003a). Structure comparison revealed that BlaB has high structural similarity with NDM-1, with an r.m.s.d of $1.5 \AA$ for all $220 \mathrm{Ca}$ atoms, which is consistent with the high amino acid sequence identity $(26 \%)$ shared by these enzymes. Superimposition of the active site structures of NDM-1 and BlaB indicates that the catalytic zinc ions and key residues for the enzymatic activity of these two MBLs are identical, suggesting similar (if not exactly the same) inhibitory mechanisms and binding modes of inhibitors and substrates.

In contrast, three other residues in the BlaB structure, which are crucial for D-captopril interaction, are not as conserved in NDM-1 (Fig. 3F). The Lys167 of BlaB stabilizes D-captopril through its $\mathrm{N}^{3}$ atom hydrogen bonding with the $\mathrm{O}_{2}$ atom of carboxylic group of D-captopril. However, the equivalent position in NDM-1 is Met114, whose $C \varepsilon$ atom has a distance $>4 \AA$ from the carboxylic group of $D$-captopril and cannot form an ideal hydrogen bond to stabilize Dcaptopril. Nevertheless, the counterpart of BlaB Asp119, whose amide main chain stabilizes D-captopril through hydrogen bonds with the $\mathrm{O}_{3}$ atom, is $\mathrm{G} \ln 123$ in NDM- 1 . Because the side chain of $G \ln 123$ is longer than that of Asp119 and sterically clashes with D-captopril, it is conceivable that the side chain of Gln123 and/or D-captopril spatially shift after NDM-1 binds D-captopril. NDM-1 Asn179, which is equivalent to Tyr233 in BlaB, forms more ideal hydrogen bonds with $\mathrm{D}$-captopril, suggesting a higher binding affinity that is consistent with the higher inhibitory effect of $D$ captopril on NDM-1 than on BlaB.

On the basis of the crystal structure of NDM-1 and the reasonable model in complex with its inhibitor D-captopril, we may be able to design compounds with high binding affinity to block the favorable interactions between NDM-1 and antibiotics. For example, extension of the carboxylic group of $\mathrm{D}$ captopril at the $\mathrm{O}_{2}$ atom will increase the interaction between NDM-1 and the new compound, thus enhancing the inhibitory effect on NDM-1 enzymatic activity.

However, several key points concerning drug discovery targeting of drug-resistant NDM-1 bacteria should be noted. First, dozens of MBLs have been identified in humans, and the currently reported compounds that inhibit the enzymatic activity of bacterial MBLs could consequently thwart the activity of human MBLs, resulting in high cytotoxicity (Lassaux et al., 2010). Second, although the reported MBLs can specifically hydrolyze their substrate antibiotics, none of them has as wide a spectrum of activity on antibiotics as NDM-1. However, the crystallographic information reported in this work reveals that the structure of NDM-1 shares high similarity with previously investigated MBLs, thus indicating similarity between their enzymatic mechanisms. Although the shape of MBL active sites is generally considered to be crucial for their substrate specificity, the substrate specificity of bacterial MBLs remains controversial and should be considered during future drug discovery efforts. Finally, while several bacteria produce MBLs related to drug resistance, none of these MBLs induces broad drugresistant infections, indicating that the intrinsic mechanism of blaNDM-1 gene transfer among different bacterial populations is essential for understanding how NDM-1 causes severe infection. Indeed, the mechanism of drug resistance by MBLs may be more complicated than we ever thought.

\section{DISCUSSION}

blaNDM-1 is a recently discovered gene that can transfer among different bacterial populations and cause severe drug resistance through its encoded NDM-1 enzyme, an MBL that hydrolyzes antibiotics. Therefore, NDM- 1 has been characterized as an attractive target to treat these drug-resistant infections. Here, we report the crystal structures of the NDM-1 enzyme, which displays the characteristic MBL family architecture, with two catalytic zinc ions in its active center. Our results indicate that NDM-1 shares structural and functional similarity with other reported MBLs. The unique features on primary sequence and unusually large cavity of the active site distinguish NDM-1 from other reported MBLs and might contribute to the wide spectrum antibiotics substrate selectivity of NDM-1. Together with mass spectroscopy and enzymatic assay analysis, D-captopril is indicated as a potential inhibitor of NDM-1 by binding to its active site. It is conceivable that novel compounds that are structurally similar to D-captopril and inhibit NDM-1 activity by competitively binding to its active center with high affinity can be used to treat super-resistant bacteria. However, while several bacterial MBLs related to drug resistance have previously been examined, none of them induces broad drug resistant bacterial infections like NDM-1, indicating that the intrinsic mechanism of blaNDM-1 gene transfer among different bacterial populations could be also essential for understanding how blaNDM-1 causes such severe infections. Nonetheless, the crystallographic and biological information reported here laid the groundwork for developing new therapeutic treatments for NDM-1-related drug-resistant bacterial infections worldwide. 


\section{MATERIALS AND METHODS}

\section{Protein expression and purification}

The gene encoding NDM-1 was chemically synthesized as reported previously (Yong et al., 2009; Kumarasamy et al., 2010). Several constructs were generated according to preliminary sequence analysis, among which the leader peptide was truncated for protein production and purification. The construct encoding residues Gly47-Arg270 (numbered as in the full length protein) of NDM-1 was ultimately chosen for final protein expression, purification, crystallization and enzymatic assays. The target gene was inserted into the pGEX-6p-1 vector (GE Healthcare) with BamHI and Xhol (bold and underlined) restriction sites using the cloning primers 5'-ACGGGATCCGGCGATCTGGTTTTCC-3' (forward) and 5'CTTCTCGAGTCAGCGCAGCTTG-3' (reverse). The accuracy of the inserts was verified by sequencing.

The recombinant plasmid was transformed into Escherichia coli strain BL21 (DE3) and over-expressed as a GST fusion protein. The cells were grown with shaking for $4-5 \mathrm{~h}$ at $37^{\circ} \mathrm{C}$ in $800 \mathrm{~mL}$ LB media containing $100 \mu \mathrm{g} / \mathrm{mL}$ ampicillin until the $\mathrm{OD}_{600}$ reached $0.4-0.6$. Then, the culture was transferred to $25^{\circ} \mathrm{C}$, and protein expression was induced for $16-20 \mathrm{~h}$ with $0.1 \mathrm{mmol} / \mathrm{L}$ isopropyl- $\beta-\mathrm{D}-1$ thiogalactopyranoside (IPTG). Harvested cells were resuspended in lysis buffer containing $50 \mathrm{mmol} / \mathrm{L}$ MES (pH 6.5), $150 \mathrm{mmol} / \mathrm{L} \mathrm{NaCl}$, and $5 \%$ glycerol and homogenized with a JN-3000 PLUS low temperature ultra-high pressure cell disrupter (JNBIO, Guangzhou). The lysate was centrifuged at $25,000 \mathrm{~g}$ for $20 \mathrm{~min}$ at $4^{\circ} \mathrm{C}$ to remove cell debris. The supernatant was then loaded twice onto a GST column pre-equilibrated with lysis buffer, and the GST tag was removed by digestion with PreScission protease overnight at $4^{\circ} \mathrm{C}$. NDM-1 was further purified by Resource $Q$ anion exchange and Superdex-75 gel filtration chromatography (GE Healthcare). Fractions were analyzed with SDS-PAGE, and the final purity was $>99 \%$. The purified protein was then concentrated to $5 \mathrm{mg} / \mathrm{mL}$ in a buffer containing $50 \mathrm{mmol} / \mathrm{L}$ MES (pH 6.5), $150 \mathrm{mmol} / \mathrm{L} \mathrm{NaCl}$, and $5 \%$ glycerol for crystallization.

\section{Crystallization}

Initial crystallization conditions were screened by the hanging drop vapor-diffusion method using commercially available Hampton Research crystal screening kits at $18^{\circ} \mathrm{C}$. Droplets containing $1 \mu \mathrm{L}$ of $5 \mathrm{mg} / \mathrm{mL}$ protein and $1 \mu \mathrm{L}$ of mother liquor were equilibrated against $200 \mu \mathrm{L}$ of reservoir solution.

Small and flaky crystals of various sizes with extensive twinning first appeared and were found to belong to the hexagonal space group in a buffer containing $20 \mathrm{mmol} / \mathrm{L} \mathrm{CC_{C }}, 20 \mathrm{mmol} / \mathrm{L} \mathrm{CaCl}$, $20 \mathrm{mmol} / \mathrm{L} \mathrm{CoCl}_{2}$ and 20\% (v/w) PEG 3350 after 1 day. However, none of these small crystals diffracted beyond $5 \AA$. Further optimization with additive and detergent screens (Hampton Research, USA) was performed, and the final optimized crystals $(200 \mu \mathrm{m} \times$ $200 \mu \mathrm{m} \times 40 \mu \mathrm{m})$ were grown in buffer containing $20 \mathrm{mmol} / \mathrm{L}$ $\mathrm{CdCl}_{2}, 20 \mathrm{mmol} / \mathrm{L} \mathrm{NaCl}, 5 \%$ PEG mono ether 550 and 25\% ( $\left./ \mathrm{w}\right)$ PEG 3350 within 3 days and diffracted to $2.0 \AA$. Precipitant in crystallization buffer was sufficient as cyro-protectant. Thus, no reagent was added before flash-cooling the crystals in a cold nitrogen stream.

\section{Data collection, processing, and structure determination}

Fresh, large crystals of native NDM-1 were harvested with a nylon loop and directly cooled in a nitrogen stream at $100 \mathrm{~K}$. The initial crystal with good diffraction quality diffracted to $2.1 \AA$ at beam line 3W1A of the Beijing Synchrotron Radiation Facility (BSRF). However, extensive twinning data generated a very high $R_{\text {merge }}$ value and were not suitable for structure determination. An optimized crystal dehydrated with $4 \mathrm{~mol} / \mathrm{L}$ sodium formate produced processable diffraction data, and a native dataset with 3-Å resolution was collected at beam line BL17U1 of the Shanghai Synchrotron Radiation Facility (SSRF). This dataset was used to generate the first model of NDM-1. The final high resolution $X$-ray diffraction data were collected at beam line NE3A of the Photon Factory (Japan) on an ADSC Q270 CCD detector at a wavelength of $1.0000 \AA$. The datasets collected at SSRF and $\mathrm{PF}$ were merged to increase resolution and data completeness. The crystal belonged to space group $P 3_{1}$, with unit-cell parameters a $=b=40.7 \AA, c=215.3 \AA$, and $\alpha=\beta=90^{\circ}, \gamma=120^{\circ}$. Assuming that there are two molecules of NDM-1 per asymmetric unit giving a Matthews coefficient of $2.2 \AA^{3} / \mathrm{Da}$ with a solvent content of $43 \%$ (Matthews, 1968). The raw data were processed with HKL2000 (Otwinowski and Minor, 1997).

The PHASER program (McCoy et al., 2007) was used to determine the correct solution via the molecular replacement method with VIM-4 (PDB code 2WRS; sharing 32\% amino acid sequence

Table 1 X-ray data collection and refinement statistics

\begin{tabular}{|c|c|}
\hline Parameters & NDM-1 \\
\hline \multicolumn{2}{|l|}{ Data collection } \\
\hline Space group & $P 3_{1}$ \\
\hline Cell parameters $\left(\AA,{ }^{\circ}\right)$ & $\begin{array}{c}a=52.5, b=72.3, c=107.3 \\
\alpha=\beta=90^{\circ}, \gamma=120^{\circ}\end{array}$ \\
\hline Wavelength $(\AA)$ & 1.0000 \\
\hline Resolution range $(\AA)^{\mathrm{a}}$ & $40.00-2.50(2.60-2.50)$ \\
\hline$R_{\text {merge }}(\%)^{\mathrm{b}}$ & $7.4(19.1)$ \\
\hline No. of all observed reflections & $21,209(8,837)$ \\
\hline No. of unique reflections & $10,177(1,280)$ \\
\hline Completeness (\%) & $71.3(87.6)$ \\
\hline $1 / \sigma(I)$ & $34.1(32.5)$ \\
\hline \multicolumn{2}{|l|}{ Refinement } \\
\hline Resolution range $(\AA)$ & $40.00-2.50$ \\
\hline No. of reflections used & 9265 \\
\hline$R_{\text {work }}(\%)$ & 23.4 \\
\hline$R_{\text {free }}(\%)^{\mathrm{c}}$ & 26.7 \\
\hline Overall average B-factor $\left(\AA^{2}\right)$ & 18.8 \\
\hline \multicolumn{2}{|l|}{ r.m.s. deviation } \\
\hline rmsd bond lengths $(\AA)$ & 0.016 \\
\hline rmsd bond angles $\left({ }^{\circ}\right)$ & 1.785 \\
\hline \multicolumn{2}{|c|}{$\begin{array}{l}\text { a Values in parentheses refer to the highest resolution shell. } \\
\text { b } R_{\text {merge }}=\Sigma \mathrm{h} \Sigma \mathrm{I} \mid \text { lih }-<\mathrm{lh}>|/ \Sigma \mathrm{h} \Sigma \mathrm{I}| \mathrm{Ih}>\text {, where }<\mathrm{Ih}>\text { is the mean of } \\
\text { multiple observations lih of a given reflection } \mathrm{h} \text {. }\end{array}$} \\
\hline \multicolumn{2}{|c|}{$\begin{array}{l}{ }^{\mathrm{c}} R_{\text {work }}=\Sigma|| \mathrm{Fp}(\mathrm{obs})|-| \mathrm{Fp}(\mathrm{calc})|| / \Sigma|\mathrm{Fp}(\mathrm{obs})| ; R_{\text {free }} \text { is an } \mathrm{R} \text {-factor for a } \\
\text { selected subset }(5 \%) \text { of reflections that was not included in prior } \\
\text { refinement calculations. }\end{array}$} \\
\hline
\end{tabular}


identity with NDM-1) (Lassaux et al., 2010) as the initial search model. Clear solutions were indicated by rotation and translation of Z-scores. Manual model building and refinement were performed with COOT (Emsley and Cowtan, 2004) and PHENIX (Adams et al., 2002). During the later stages of positional refinement, restraints were relaxed, and a bulk solvent correction was applied under the guidance of $R_{\text {free. }}$. Model geometry was verified using the program PROCHECK (Laskowski et al., 1993). Solvent molecules were located from stereochemically reasonable peaks in the $\sigma A$-weighted $2 F o-F C$ difference electron density map at $1.2 \sigma$ in COOT. Final refinement statistics are summarized in Table 1. Structural figures were drawn with PyMOL (DeLano, 2002).

\section{Enzymatic assay}

NDM-1 enzymatic activity was monitored with a spectrophotometric assay, using imipenem as the substrate to transfer the action of the NDM-1 to an observable decrease in the absorption of imipenem at $300 \mathrm{~nm}$. To measure the enzymatic activity of wild-type NDM-1 and relevant mutants, assays were conducted based upon the activity of NDM-1 observed above. Briefly, $50 \mathrm{mmol} / \mathrm{L}$ HEPES (pH 7.3), $10 \mu \mathrm{g} / \mathrm{mL}$ BSA, $500 \mu \mathrm{mol} / \mathrm{L}$ imipenem, and $50 \mathrm{nmol} / \mathrm{L} \mathrm{NDM}-1$ protein were mixed in a total volume of $1 \mathrm{~mL}$. The reaction mixture was incubated at $37^{\circ} \mathrm{C}$, and the $A_{300}$ value was immediately measured using a GENE Quant 1300 spectrometer (GE Healthcare). To determine the inhibitory effect of ethylenediaminetetraacetic acid (EDTA) and L/D-captopril, these reagents were added to the reaction buffer at various concentrations.

\section{ESI-MS analysis of NDM-1 binding with inhibitor}

NDM-1 protein was buffer-exchanged into $10 \mathrm{mmol} / \mathrm{L}$ ammonium acetate $(\mathrm{pH}$ 7.0) for mass spectrometry (MS). D-captopril was first dissolved in $\mathrm{ddH}_{2} \mathrm{O}$ to create an $80-\mathrm{mmol} / \mathrm{L}$ stock solution and further diluted with $10 \mathrm{mmol} / \mathrm{L}$ ammonium acetate $(\mathrm{pH} \mathrm{7.0)}$. The final concentrations of the protein and the inhibitor during incubation at room temperature for $10 \mathrm{~min}$ were $10 \mu \mathrm{mol} / \mathrm{L}$ and $50 \mu \mathrm{mol} / \mathrm{L}$, respectively. The reaction was then loaded into an off-line nanospray tip (Waters, UK) prior to MS analysis.

All analyses were performed on a Synapt Q-IM-TOF mass spectrometer (Waters, UK) equipped with a nano-ESI ion source. The major instrument settings were as follows: spray voltage $1000-1200 \mathrm{~V}$, cone voltage $30 \mathrm{~V}$, source block temperature $45^{\circ} \mathrm{C}$, and nano gas flow-rate $0.1-0.3 \mathrm{~L} / \mathrm{h}$. Full-scan mass spectra of positive ions were recorded in the profile mode, covering the mass range of $1000-4500 \mathrm{~m} / \mathrm{z}$. All data were processed and transformed into the actual mass scale using the MassLynx software 4.1 from Waters.

\section{ACKNOWLEDGEMENTS}

We are grateful for the help of the beam staffs from the Beijing Synchrotron Radiation Facility, Shanghai Synchrotron Radiation Facility, and Photon Factory. We also thank Drs. Z. Miao, B. Sun and W. Li for their generous help with chemical compound synthesis and mass spectrum analysis.

This work was supported by the National Natural Science Foundation of China (Grant Nos. 30870486 and 30730022), the National Major Projects (Grant Nos. 2009ZX10004-804,
2009ZX09311-001 and 2009ZX10004-304) and the National Basic Research Program (973 Program) (Grant Nos. 2011CB915501 and 2011CB910304).

\section{ABBREVIATIONS}

AUC, analytical ultracentrifugation; EDTA, ethylenediaminetetraacetic acid; IPTG, isopropyl- $\beta$-D-1-thiogalactopyranoside; MBL, metallo$\beta$-lactamase; NDM-1, New Delhi metallo- $\beta$-lactamase 1

\section{REFERENCES}

Abraham, E.P., and Chain, E. (1988). An enzyme from bacteria able to destroy penicillin. 1940. Rev Infect Dis 10, 677-678.

Adams, P.D., Grosse-Kunstleve, R.W., Hung, L.W., loerger, T.R., McCoy, A.J., Moriarty, N.W., Read, R.J., Sacchettini, J.C., Sauter, N.K., and Terwilliger, T.C. (2002). PHENIX: building new software for automated crystallographic structure determination. Acta Crystallogr D Biol Crystallogr 58, 1948-1954.

Antony, J., Gresh, N., Olsen, L., Hemmingsen, L., Schofield, C.J., and Bauer, R. (2002). Binding of D- and L-captopril inhibitors to metallobeta-lactamase studied by polarizable molecular mechanics and quantum mechanics. J Comput Chem 23, 1281-1296.

Baiden, F., Owusu-Agyei, S., Webster, J., and Chandramohan, D. (2010). The need for new antibiotics. Lancet 375, 637-638.

Bauernfeind, A., Chong, Y., and Lee, K. (1998). Plasmid-encoded AmpC beta-lactamases: how far have we gone 10 years after the discovery? Yonsei Med J 39, 520-525.

Bebrone, C. (2007). Metallo-beta-lactamases (classification, activity, genetic organization, structure, zinc coordination) and their superfamily. Biochem Pharmacol 74, 1686-1701.

Carfi, A., Pares, S., Duée, E., Galleni, M., Duez, C., Frère, J.M., and Dideberg, O. (1995). The 3-D structure of a zinc metallo-betalactamase from Bacillus cereus reveals a new type of protein fold. EMBO J 14, 4914 4921.

Chihara, S., Okuzumi, K., Yamamoto, Y., Oikawa, S., and Hishinuma, A. (2011). First case of New Delhi metallo-beta-lactamase 1producing Escherichia coli infection in Japan. Clin Infect Dis 52, 153-154.

Concha, N.O., Janson, C.A., Rowling, P., Pearson, S., Cheever, C.A., Clarke, B.P., Lewis, C., Galleni, M., Frère, J.M., Payne, D.J., et al. (2000). Crystal structure of the IMP-1 metallo beta-lactamase from Pseudomonas aeruginosa and its complex with a mercaptocarboxylate inhibitor: binding determinants of a potent, broadspectrum inhibitor. Biochemistry 39, 42884298.

Daiyasu, H., Osaka, K., Ishino, Y., and Toh, H. (2001). Expansion of the zinc metallo-hydrolase family of the beta-lactamase fold. FEBS Lett $503,1-6$.

DeLano, W. (2002). The PyMOL Molecular Graphics System. San Carlos, CA: DeLano Scientic.

Emsley, P., and Cowtan, K. (2004). Coot: model-building tools for molecular graphics. Acta Crystallogr D Biol Crystallogr 60, 2126-2132.

Garau, G., Bebrone, C., Anne, C., Galleni, M., Frère, J.M., and Dideberg, O. (2005). A metallo-beta-lactamase enzyme in action: crystal structures of the monozinc carbapenemase $\mathrm{CphA}$ and its complex with biapenem. J Mol Biol 345, 785-795.

Garcia-Saez, I., Docquier, J.D., Rossolini, G.M., and Dideberg, O. (2008). The three-dimensional structure of VIM-2, a Zn-beta- 
lactamase from Pseudomonas aeruginosa in its reduced and oxidised form. J Mol Biol 375, 604-611.

García-Saez, I., Hopkins, J., Papamicael, C., Franceschini, N., Amicosante, G., Rossolini, G.M., Galleni, M., Frère, J.M., and Dideberg, O. (2003a). The 1.5-A structure of Chryseobacterium meningosepticum zinc beta-lactamase in complex with the inhibitor, D-captopril. J Biol Chem 278, 23868-23873.

García-Sáez, I., Mercuri, P.S., Papamicael, C., Kahn, R., Frère, J.M., Galleni, M., Rossolini, G.M., and Dideberg, O. (2003b). Threedimensional structure of FEZ-1, a monomeric subclass B3 metallobeta-lactamase from Fluoribacter gormanii, in native form and in complex with D-captopril. J Mol Biol 325, 651-660.

Heddini, A., Cars, O., Qiang, S., and Tomson, G. (2009). Antibiotic resistance in China-a major future challenge. Lancet 373, 30.

Krissinel, E., and Henrick, K. (2004). Secondary-structure matching (SSM), a new tool for fast protein structure alignment in three dimensions. Acta Crystallogr D Biol Crystallogr 60, 2256-2268.

Kumarasamy, K.K., Toleman, M.A., Walsh, T.R., Bagaria, J., Butt, F., Balakrishnan, R., Chaudhary, U., Doumith, M., Giske, C.G., Irfan, S., et al. (2010). Emergence of a new antibiotic resistance mechanism in India, Pakistan, and the UK: a molecular, biological, and epidemiological study. Lancet Infect Dis 10, 597-602.

Laskowski, R., MacArthur, M., Moss, D., and Thornton, J. (1993). PROCHECK: a program to check the stereochemical quality of protein structures. J Appl Cryst 26, 283-291.

Lassaux, P., Hamel, M., Gulea, M., Delbrück, H., Mercuri, P.S., Horsfall, L., Dehareng, D., Kupper, M., Frère, J.M., Hoffmann, K., et al. (2010). Mercaptophosphonate compounds as broad-spectrum inhibitors of the metallo-beta-lactamases. J Med Chem 53, $4862-4876$.

Livermore, D.M. (2009). Has the era of untreatable infections arrived? J Antimicrob Chemother 64, i29-i36.

Matthews, B.W. (1968). Solvent content of protein crystals. J Mol Biol 33, 491-497.

McCoy, A.J., Grosse-Kunstleve, R.W., Adams, P.D., Winn, M.D., Storoni, L.C., and Read, R.J. (2007). Phaser crystallographic software. J Appl Crystallogr 40, 658-674.

Moali, C., Anne, C., Lamotte-Brasseur, J., Groslambert, S., Devreese, B., Van Beeumen, J., Galleni, M., and Frère, J.M. (2003). Analysis of the importance of the metallo-beta-lactamase active site loop in substrate binding and catalysis. Chem Biol 10, 319-329.

Moellering, R.C. Jr. (2010). NDM-1-a cause for worldwide concern. N Engl J Med 363, 2377-2379.

Otwinowski, Z., and Minor, W. (1997). Processing of X-ray diffraction data collected in oscillation mode. In: Macromolecular Crystallography, part A. Carter C.W. Jr., and Sweet R.M., eds. New York: Academic Press. 307-326.

Sabbath, L.D., and Abraham, E.P. (1966) Zinc as a cofactor for cephalosporinase from Bacillus cereus 569. Biochem J 98, 11c-13c.

Shimada, A., Ishikawa, H., Nakagawa, N., Kuramitsu, S., and Masui, R. (2010). The first crystal structure of an archaeal metallo-betalactamase superfamily protein; ST1585 from Sulfolobus tokodaii. Proteins 78, 2399-2402.

Struelens, M.J., Monnet, D.L., Magiorakos, A.P., Santos O'Connor, F., and Giesecke, J., and the European NDM-1 Survey Participants. (2010). New Delhi metallo-beta-lactamase 1-producing Enterobacteriaceae: emergence and response in Europe. Euro Surveill 15, pii $=19716$.

Walsh, T.R. (2010). Emerging carbapenemases: a global perspective. Int J Antimicrob Agents 36, S8-S14.

Walsh, T.R., Weeks, J., Livermore, D.M., and Toleman, M.A. (2011). Dissemination of NDM-1 positive bacteria in the New Delhi environment and its implications for human health: an environmental point prevalence study. Lancet Infect Dis 11, 355-362

Yong, D., Toleman, M.A., Giske, C.G., Cho, H.S., Sundman, K., Lee, K., and Walsh, T.R. (2009). Characterization of a new metallo-betalactamase gene, bla(NDM-1), and a novel erythromycin esterase gene carried on a unique genetic structure in Klebsiella pneumoniae sequence type 14 from India. Antimicrob Agents Chemother $53,5046-5054$. 